\title{
Nachrichten
}

Formular-Management für interaktive eGovernment-Dienste

Die Landesverwaltung Rheinland-Pfalz hat sich für das ganzheitliche Formular-Management-System der bol Behörden Online Systemhaus $\mathrm{GmbH}$ in München entschieden. Ziel der Ausschreibung war es, ein Formular-Management-System zu beschaffen, das modernsten Standards genügt und die webbasierte Verwendung von externen und internen elektronischen Formularen ermöglicht. Neben dem Erstellen, Publizieren, Ausfüllen, Speichern, Signieren, Verschlüsseln und Versenden durch den Nutzer steht vor allem die medienbruchfreie Annahme und Bereitstellung der Daten zur Weiterverarbeitung in den Behörden im Vordergrund.

Die Integration in bestehende Anwendungen wie beispielsweise DOMEA, Fachverfahren und Internetplattformen sind ebenso wichtige Voraussetzung wie die einfache Nutzung der Anwendung ohne zusätzliche Software-Komponenten auf der Anwenderseite. Um die Servicequalität und Akzeptanz zu erhöhen, ist beispielsweise auch die Vorbelegung von eFormularen mit Daten wie zum Beispiel Stammdaten angedacht. Auch die Unterbrechung und spätere Fortsetzung der Antragsbearbeitung oder das Anhängen von digitalisierten Anlagen zu eFormularen sind Funktionen, die für den Nutzer eine Vereinfachung in der Kommunikation mit den Behörden bedeuten.

Weitere Informationen: bol Behörde Online Systemhaus GmbH, Oliver Wolf, Hubertusstraße 4, 80639 München, Telefon: (089) 21096715, E-Mail: Oliver.Wolf @bol-systemhaus.de, Internet: www.behoerden-online.de.

\section{Kommunales Vereinsleben}

Vereine sind das «Salz in der Suppe« eines jeden Gemeinwesens. Aber wie stark prägen sie deren Leben? In welchen Bereichen engagieren und wie finanzieren sie sich? Diesen Fragen ist die Münsteraner Professorin Dr. Annette Zimmer nachgegangen. Wie das Team des Zentrums für Nonprofit-Management herausgefunden hat, ist von mindestens 1.600 aktiven Gruppen auszugehen. Und darin sind noch nicht einmal Initiativen enthalten, die nicht als »e.V.«geführt werden.

Die Auswertung zeigt eine hoch differenzierte und komplexe Vereinslandschaft. Die Szene ist von relativ kleinen Vereinigungen geprägt: Die Mehrheit hat eine Mitgliederbasis von unter 100 Personen. Doch die Bedeutung der jeweiligen Gruppe ergibt sich nicht allein aus ihrer Mitgliederzahl und der Vielfalt ihrer Aktivitäten. Ein wichtiger Indikator für die Bedeutung des Vereinswesens ist auch die beachtliche Finanzkraft. Allerdings sind die Mittel sehr ungleich verteilt. Die überwiegende Mehrheit der Vereinigungen ist hinsichtlich ihrer Finanzkraft eher klein: Zwei Drittel haben weniger als 20.000 Euro pro Jahr zur Verfügung.

Mehr im Internet unter www.npm-online.de/muenster-engagiert.

\section{Bistum mit modernem Finanzmanagement und Berichtswesen}

Das Bistum Aachen hat der MACH AG den Zuschlag für die Einführung einer neuen Finanzsoftware erteilt. Damit nutzen künftig bereits acht von insgesamt 27 (Erz-)Bistümern in Deutschland diese Software, um ihr Finanzmanagement neu auszurichten. Der Marktanteil von $\mathrm{MACH}$ im Bereich der katholischen Bistümer in Deutschland erhöht sich auf rund 30 Prozent.

Das Bistum Aachen will angesichts schwieriger Rahmenbedingungen - sinkende Kirchensteuereinnahmen und wachsender Kostendruck - die Aussagekraft seines Finanz- und Rechnungswesens erhöhen. Deswegen hat sich die Diözesanverwaltung ent- schlossen, die IT-Strukturen zu modernisieren und mit einer neuen Software für das Finanz- und Verwaltungsmanagement die kaufmännische Buchführung einzuführen. Das Bistum will so Kostentransparenz und Kostenbewusstsein erhöhen und ein umfassendes Controllingsystem etablieren, um seine seelsorgerischen Aufgaben besser erfüllen zu können.

Weitere Informationen: MACH AG, Jochen Michels, Wielandstraße 14, 23558 Lübeck, Telefon: (0451) 70647-271, E-Mail: jmi@mach.de, Internet: www.mach.de.

\section{Datenbank für Verwaltungsvorschriften des Bundes online}

Zahlreiche Verwaltungsvorschriften des Bundes sind für die Behörden künftig schnell und einfach per Mausklick abrufbar in der neuen Datenbank für Verwaltungsvorschriften des Bundes DB VwV Bund. Ab dem 1. März 2005 steht den Beschäftigten der Bundesverwaltung ein modernes elektronisches Arbeits- und Informationsmittel zur Verfügung, über das sie zunächst die rund 580 gültigen Verwaltungsvorschriften des Bundesministeriums des Innern abrufen können. Dabei wurde nicht nur der Zugang zu den Vorschriften modernisiert. Gleichzeitig wurden die Vorschriften gründlich durchforstet - mit Erfolg: 20 Prozent der Vorschriften des BMI wurden gestrichen.

Die Datenbank verknüpft die eingestellten Verwaltungsvorschriften elektronisch mit dem zu Grunde liegenden Bundesrecht und der maßgebenden Rechtsprechung. Der gesamte Rechtshintergrund zu den jeweiligen Vorschriften wird so übersichtlich und »auf einen Klick « transparent. Dadurch trägt die Datenbank auch zu größerer Rechtssicherheit und Qualität in der Anwendung der Verwaltungsvorschriften des Bundes bei.

Das Gesamtvorhaben - mit der Beteiligung aller Ressorts - soll im kommenden Jahr realisiert werden. In Zukunft werden auch Interessierte aus den Länderverwaltungen und der Wirtschaft mit gestaffelten Zugangsmöglichkeiten die Datenbank nutzen können. Auch Bürgerinnen und Bürger werden die Möglichkeit bekommen, auf die Datenbank für Verwaltungsvorschriften des Bundes zuzugreifen.

\section{Bundesagentur für Arbeit setzt auf ITIL-Lösung}

Das MATERNA IT-Service-Management-Framework (ITSM-Framework) ist bei der Bundesagentur für Arbeit (BA) im Einsatz. Die Bundesagentur für Arbeit steuert über ihr zentrales Rechenzentrum rund 200 dezentrale Rechenzentren im gesamten Bundesgebiet. Sie betreibt mit ihren über 100.000 PC-Arbeitsplätzen und der zugehörigen Infrastruktur eine der größten IT-Landschaften weltweit. Der reibungslose IT-Betrieb setzt voraus, dass alle Änderungen an der IT-Infrastruktur professionell durchgeführt werden und alle ITKomponenten in ihren Abhängigkeiten bekannt sind. Seit kurzem unterstützt das ITIL-basierte MATERNA ITSM-Framework erste wichtige IT-Prozesse in der Behörde. Implementiert wurden die Module Change und Configuration Management.

Weitere Informationen: MATERNA GmbH, Christine Siepe, Voßkuhle 37, 44141 Dortmund, Telefon: (0231) 5599-168, E-Mail: Christine.Siepe@Materna.de, Internet: www.materna.de/ presse.

\section{Baden-Württemberg als Open-Source-Standort}

Baden-Württemberg setzt auf den Ausbau seines Open-SourceClusters: Die MFG Baden-Württemberg, das Kompetenzzentrum des Landes für IT und Medien, baut die führende Position mit einem umfassenden Maßnahmenpaket weiter aus. Im Mittelpunkt steht die Schaffung von Wettbewerbsvorteilen und Arbeitsplätzen 


\section{Nachrichten}

durch die Nutzung von Open-Source-Software in Forschung, Wirtschaft und Verwaltung. In Stuttgart wurde die Linux Solutions Group (LiSoG) gegründet, an der IT-Unternehmen aus dem gesamten deutschsprachigen Raum wie IBM, MySQL, Novell, Siemens Business Services und RedHat sowie Universitäten und Anwender beteiligt sind. Ziel ist es, den Einsatz von linuxbasierten Lösungen zu fördern. Die MFG wurde mit der Geschäftsführung beauftragt. Zeitgleich hat Hewlett-Packard die MFG beauftragt, die bundesweite Initiative »Linux Kommunale« zu betreuen, bei der es um den effektiven Einsatz von Open Source im Public Sector geht.

Weitere Informationen: MFG Baden-Württemberg, Kompetenzzentrum des Landes für IT und Medien, Jennifer Bader, Telefon: (0711) 90715-316, E-Mail: bader@mfg.de

\section{Kabinettbeschluss zu BundOnline}

Das Bundeskabinett hat mit dem Beschluss zu BundOnline die Grundlagen für eine dauerhafte Finanzierung der aufgebauten eGovernment-Infrastruktur geschaffen. Damit sind die Ergebnisse der Initiative über 2005 hinaus gesichert. Zur langfristigen Sicherung der getätigten Investitionen sind die Bundes- behörden künftig verpflichtet, die BundOnline-Basiskomponenten (virtuelle Poststelle mit den Funktionen Signatur und Verschlüsselung, Zahlungsverkehrsplattform, Content Management System und Formularmanagementsystem) umfassend zu nutzen. Alle Bundesbehörden werden künftig ihre Behördendaten wie Kurzbeschreibung, Adresse, Telefon und Internetadresse im Portal www.bund.de einstellen. Außerdem werden ihre eGovernment- Dienstleistungen im Portal verlinkt und grundsätzlich Formulare, Stellenangebote, Ausschreibungen und Veräußerungen im Service-Center des Portals bekannt gemacht. Das bei BundOnline entwickelte Dienstleistungsportal www.bund.de wird damit als zentraler Einstiegspunkt für alle relevanten Behördeninformationen im Internet gestärkt.

\section{Zentraler Sperr-Notruf 116116}

Bundesinnenminister Otto Schily betonte auf der CeBIT die große Bedeutung der zentralen Sperr-Notrufnummer 116116 als schneller Anlaufpunkt nach Diebstahl oder Verlust von Bankkarten, Kundenkarten, Firmenausweisen, Handys u.a.. Allerdings lebe diese Initiative von der Beteiligung der Wirtschaft. Der Bundesinnenminister ruft alle Unternehmen auf, sich dem Sperr-Notruf 116 116 anzuschließen, um einen effektiven Schutz zu gewährleisten.

\section{Doch »papierloses« Büro ?}

Jedenfalls wird im saarländischen Umweltministerium deutlich weniger Papier verbraucht als traditionell. Immerhin ging der Papierverbrauch allein in diesem Ressort von knapp 2,4 Millionen Blatt in 2003 auf 1,3 Millionen in 2004 zurück. Die Gründe: beidseitiges Kopieren und Drucken sowie konsequente Nutzung von elektronischer Post Intranet. (Nota bene: bei je zehn Ressorts in sechzehn Bundesländern summiert sich eine solche Einsparung schon auf 160 Millionen Blatt Papier - jedes Jahr).

\section{Bundeskabinett beschließt gemeinsame eCard-Strategie}

Das Bundeskabinett hat Eckpunkte für eine gemeinsame eCard Strategie der Bundesregierung zur Unterstützung der flächendeckenden Einführung von elektronischen Karten beschlossen. Vorgesehen ist, dass die geplanten Kartenprojekte der Bundesverwaltung die Elektronische Gesundheitskarte, der Digitale Personalausweis, das JobCard-Verfahren und die Elektronische Steuerer- klärung eng aufeinander abgestimmt werden. Gleiche Standards und die breite Verwendbarkeit der Chipkarten für den elektronischen Geschäftsverkehr sollen Effizienzgewinne und Kosteneinsparungen zum Nutzen von Bürgerinnen und Bürgern, Wirtschaft und Verwaltung gewährleisten. Ferner werden durch die Eckpunkte die elektronische Authentisierung (Identifizierung des Nutzers) und die qualifizierte elektronische Signatur (Äquivalent zur manuellen Unterschrift) zur Verwendung auf den Chipkarten vereinheitlicht.

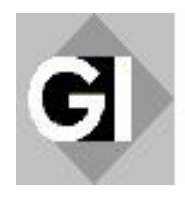

\section{Risikoanalyse vor Einführung der neuen Gesundheitskarte}

Die elektronische Gesundheitskarte bietet Patienten und Leistungserbringern große Chancen. Gleichzeitig dürfen aber mögliche Risiken nicht verharmlost werden, sondern bedürfen einer sorgfältigen Analyse insbesondere im Hinblick auf den Datenschutz und das informationelle Selbstbestimmungsrecht des Einzelnen. $\mathrm{Zu}$ diesem Schluss sind Fachleute aus Wissenschaft und Praxis auf einem von der Gesellschaft für Informatik e.V. (GI) und der Informationstechnischen Gesellschaft im VDE (ITG) organisierten Forum auf der CeBIT gekommen.

»Die Gesundheitskarte darf insbesondere im Hinblick auf das Vertrauen in die Informationstechnik in Deutschland nicht zu einem ähnlichen Desaster wie der erste Versuch von Toll Collect werden «, warnte GI-Präsident Matthias Jarke in Hannover. Dazu gehörten in einer differenzierten Risikoanalyse sowohl die sorgfältige technische Vorbereitung des Mammutprojektes als auch eine damit einhergehende detaillierte Aufklärung der Bevölkerung. Insbesondere vertrauenswürdige Datenschutzmechanismen und der selbstbestimmte Umgang mit den eigenen Daten seien die Grundvoraussetzungen für das Gelingen des Projektes.

Sofern datenschutzrechtliche Aspekte bei Zugriff und Speicherung der personenbezogenen Daten geklärt seien, böte die Gesundheitskarte große Chancen bei der Reform des Gesundheitswesens, so Jarke. Dies gelte sowohl in wirtschaftlicher wie in versorgungstechnischer Hinsicht.

Weitere Hintergrundinformationen hierzu in einem Thesenpapier unter www.gi-ev.de/download/gi-thesen-gesundheitskarte2005.pdf.

\section{Vorschau auf die kommenden Hefte}

Gerhard Hammerschmid und Werner Wutscher: Status der Verwaltungsreform in Österreich

Rolf Mohr: Kulturwechsel - Unerwartet Anstößiges zur Reform des Öffentlichen Dienstes

Georg Harle: Einführung eines kaufmännischen Rechnungswesens in Hessen

Hiltrud Naßmacher: Investition statt Konsum

Wolfgang Pippke und Bernd Klümper : Umweltschutz im Neuen Steuerungsmodell

Klaus Wirth: Moderne Gemeindeverwaltung zwischen

Kundenorientierung und Bürgerengagement

Krzysztof Borodako: Informations- und Promotions-Aktivitäten der polnischen Kreise

Martin Franz: Kollegiale Beratung-effizientes Netzwerk für die Führungspraxis

Sandra Kohl: Das Mitarbeiterinnen- und Mitarbeiterportal der Freien Hansestadt Bremen

Bodo Wiegand: Lean Management in der Kommunalverwaltung Axel Thomas: Risikomanagement im Public Leasing Geschäft Jens Seeboth: Die einheitliche Identifikationsnummer für die Wirtschaft 